\title{
Presencia y mecenazgo español en la Florencia Medicea del Quinientos
}

\author{
Blanca GonZÁlez TALAVERA \\ Departamento de Historia del Arte \\ Universidad de Granada. \\ blancagt@ugr.es
}

\section{RESUMEN}

En este artículo se analizan las principales manifestaciones artísticas de la comunidad española de la Florencia medicea del Quinientos. Para ello será necesario partir del estudio de la realidad política, social, económica y cultural de la que fueron partícipes y que permitió a los españoles alzarse como la comunidad "extranjera" más poderosa de la Florencia del Renacimiento.

Palabras clave: Florencia; España; Médicis; siglo XVI; manifestaciones artísticas.

\section{Spanish presence and patronage in Medici's Florence in the 16th century}

\begin{abstract}
This paper aims at analysing the main artistic representations of the Spanish community in Medici's Florence in the 16th century. For this purpose, it is necessary to start considering the political, social, economic and cultural reality, which surrounded them and allowed the Spaniards to arise as the most powerful "foreign" community of the Renaissance Florence.
\end{abstract}

Key words: Florence; Spain; Medici; 16th century; artistic manifestations. 
La Florencia Medicea del Quinientos fue testigo del auge de una sólida comunidad española que adquirió y mantuvo un rango social, económico, artístico y cultural que, hasta ahora, ha pasado inadvertido para la crítica. Es así como se explica la línea de investigación en la que trabajo y que ha dado como primer resultado mi tesis doctoral: "Presencia y mecenazgo español en la Florencia Medicea del Quinientos: de Cosme I a Fernando I", un trabajo más que justificado dada la carencia de estudios que sirvan de base historiográfica para el estudio de la producción artística de esta comunidad de españoles.

La fuerte presencia española debe entenderse en el ámbito del protectorado que la monarquía hispánica ejerció sobre la capital toscana a lo largo del siglo XVI, una circunstancia que favoreció la puesta en marcha del ducado florentino (más tarde Gran Ducado de Toscana), un recio aparato estatal gobernado por la familia Médicis ${ }^{1}$. Éste no habría tenido lugar sin la tutela española o, al menos, no habría gozado de la sólida configuración que alcanzó desde Cosme I hasta Fernando I de Médicis (1537-1609).

Uno de los factores determinantes en el rápido ascenso del poder español en Florencia fue la política matrimonial puesta en marcha por Carlos V. Siguiendo de cerca las directrices establecidas por sus abuelos, el Emperador vio en Florencia un importante enclave desde el que poder extender la hegemonía española en Italia. De este modo, Carlos V concertó el matrimonio de su hija, Margarita de Austria, con el primer duque de Florencia, Alejandro de Médicis². Apoyó igualmente el enlace entre Cosme I de Médicis y Leonor de Toledo ${ }^{3}$, hija de Don Pedro Álvarez de Toledo, Virrey de Nápoles.

La ambivalente política exterior de los grandes duques de Toscana (entre Francia y España) no impidió la presencia de la comunidad española en Florencia, sino todo lo contrario: facilitó su asentamiento y esplendor durante todo el siglo XVI y bien entrado el siglo XVII. A partir del matrimonio Toledo-Médicis ${ }^{4}$ (fig. 1), las relacio-

1 Sobre la reposición de los Médicis en Florencia véanse los trabajos de CADENAS Y VICENT, Vicente de, El Saco de Prato, la primera reposición de los Médicis en Florencia y la presencia de España en el Milanesado: España en Italia, Madrid, Instituto «Salazar y Castro» (C.S.I.C.), 1982; además: El fin de la República florentina: segunda reposición de los Médicis en Florencia por los ejércitos españoles, Madrid, Instituto «Salazar y Castro», 1976.

2 ROMERO, Eladi, “Alejandro de Medici I duque de Florencia”, en Historia 16, n 377, 2007, pp.110-127.

3 Sobre la figura de Leonor de Toledo existe una extensa bibliografía. Fundamentales son las siguientes aportaciones: MARCOLIN, Massimo y PACETTI, Paola, "I quartieri di Cosimo I e di Eleonora di Toledo nel Palazzo Ducale dal 1540 - 1562", en Medicea, n 6, 2010, pp. 20-31; GÁLDY, Andrea, "L'appartamento di Eleonora di Toledo in Palazzo Vecchio: la scena della nuova Isabella la Cattolica", en CALVI, Giulia y SPINELLI, Riccardo (ed.), Le donne Medici nel sistema europeo delle corti: XVI - XVIII secolo, vol. II, Firenze, Edizioni Polistampa, 2008, pp. 615-626; GÁLDY, Andrea, "Tuscan concerns and Spanish heritage in the decoration of Duchess Eleonora's apartment in the Palazzo Vecchio", en Renaissance studies, n 20, 2006, pp. 293-319; HOPE, Ilaria, "A duchess' place at court: the Quartiere di Eleonora in the Palazzo della Signoria in Florence", en EISENBICHLER, Konrad (ed.), The cultural world of Eleonora die Toledo: Duchess of Florence and Siena, Aldershot, Ashgate, 2004, pp. 98-118; EDELSTEIN, Bruce, "Bronzino in the service of Eleonora di Toledo and Cosimo I de' Medici: conjugal patronage and the painter-courtier", en REISS, Sheryl E. y WILKINS, David G. (eds.), Beyond Isabella: secular women patrons of art in Renaissance Italy, Kirksville, Truman State University Press, 2001, pp. 225-261.

4 HERNANDO SÁNCHEZ, Carlos José, "Los Médicis y los Toledo: Familia y lenguaje del poder en la Italia de Felipe II", en DI STEFANO, Giuseppe; FASANO GUARINI, Elena y MARTINEGNO, Alessandro 


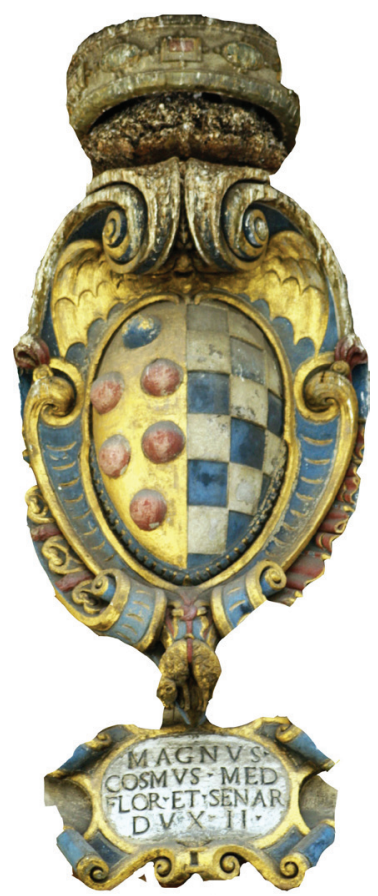

Fig. 1. Giorgio Vasari, Alianza Médicis-Toledo (detalle), ca.1567, Loggia del Pesce, Piazza dei Ciompi (Florencia).

nes entre el Rey y la familia Álvarez de Toledo adquieren un matiz significativo ya que justifican, desde el punto de vista político-diplomático, la buena gestión en los vínculos de la comunidad española de Florencia con la Casa Médicis durante toda la centuria. Además, la consecuencia directa de este enlace fue la llegada de un poderoso séquito de españoles que, acompañando a Leonor desde Nápoles a Florencia, fijó su residencia definitivamente en la capital toscana.

De este modo, la llegada de Leonor de Toledo a la capital toscana en 1539 sentó las bases de la protección medicea sobre aquellos españoles que, a partir de ese momento, desempeñaron puestos destacados en las principales arterias del estado florentino (corte, milicia, religión, universidad y mercado).

Junto con el mercado, los puestos más destacados de la comunidad española en Florencia los ocupan aquellos que desempeñan cargos relevantes en la incipiente corte ducal. Los grandes cortesanos tomaron protagonismo como nueva clase dirigente en el ámbito político, social, y, también, en el campo artístico como mecenas de las artes. Por entonces, la ciudad del Arno disfrutaba de uno de los momentos más brillantes dentro del panorama pictórico italiano. Del más puro Manierismo, con Rosso,

(coord.), Italia non spagnola e monarchia spagnola tra'500 e'600. Politica, cultura e letteratura, (Convegno Internazionale di Studi, Pisa, 1998), Florencia, Olschki, 2009. 
Pontormo, Bronzino y Carlo Portelli, la paleta florentina se tornó más "decorosa" tras los dictados de Trento, un acontecimiento que marcará un antes y un después en la pintura, dando inicio, a partir de 1563, a la "reforma florentina" de mano de Santi di Tito, al que seguirán de cerca Bernardino Poccetti, Alessandro Allori, Alessandro Fei y Giovan Maria Butteri. Un panorama que despide el Cinquecento con nuevos aires romanos introducidos, entre otros, por Cigoli, que "renueva" la pintura florentina adelantándola al espíritu barroco.

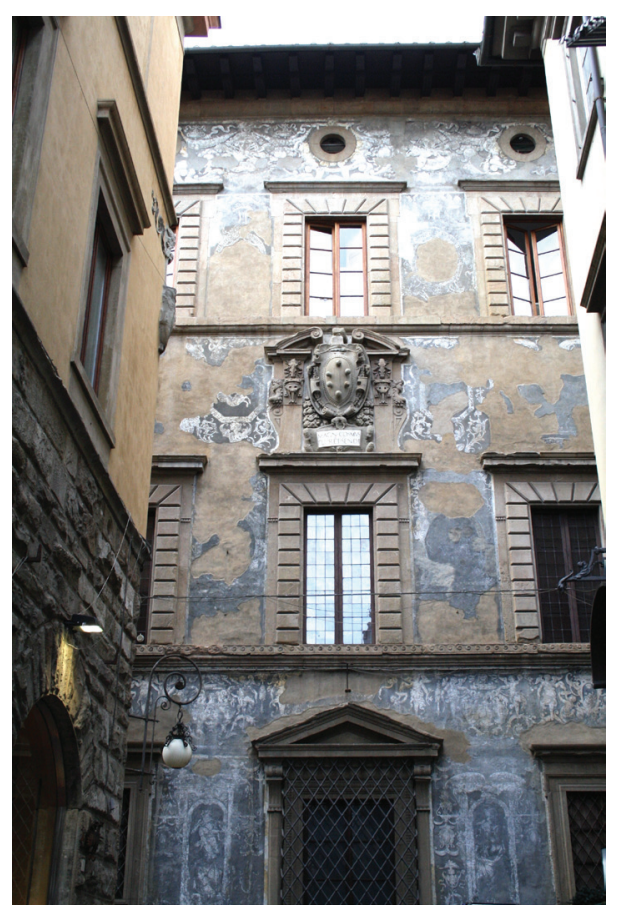

Fig. 2. Bartolommeo Ammannati y Giorgio Vasari, Fachada del Palacio Ramírez de Montalvo, Borgo degli Albizi (Florencia), segunda mitad del siglo XVI.

El personaje español más destacado de cuantos se asientan en Florencia a partir del matrimonio en 1539 de Eleonora de Toledo es, sin duda, Antonio Ramírez de Montalvo ${ }^{5}$. Todavía hoy, la ciudad del Arno recuerda el esplendor del cortesano favorito de Cosme I de Médicis: su palacio en Borgo degli Albizi (fig. 2), la Villa Montalvo de Campi Bisanzio o la capilla patronímica (en el antiguo convento de Santa Maria degli Angeli de Florencia) son tan sólo algunos vestigios de tan singular personaje. La clave de su éxito estuvo en el cumplimiento de un rapidísimo cursus honorum en el que Don Antonio se convirtió en longa manus de Cosme I de Médicis, a cuyo

5 DEL GRATTA, Rodolfo, "Antonio Ramirez Montalvo: Uno spagnolo alla Corte di Cosimo I", en Toscana e Spagna nel secolo XVI. Miscellanea di studi storici, Pisa, ETS, 1996, pp. 223-271. 


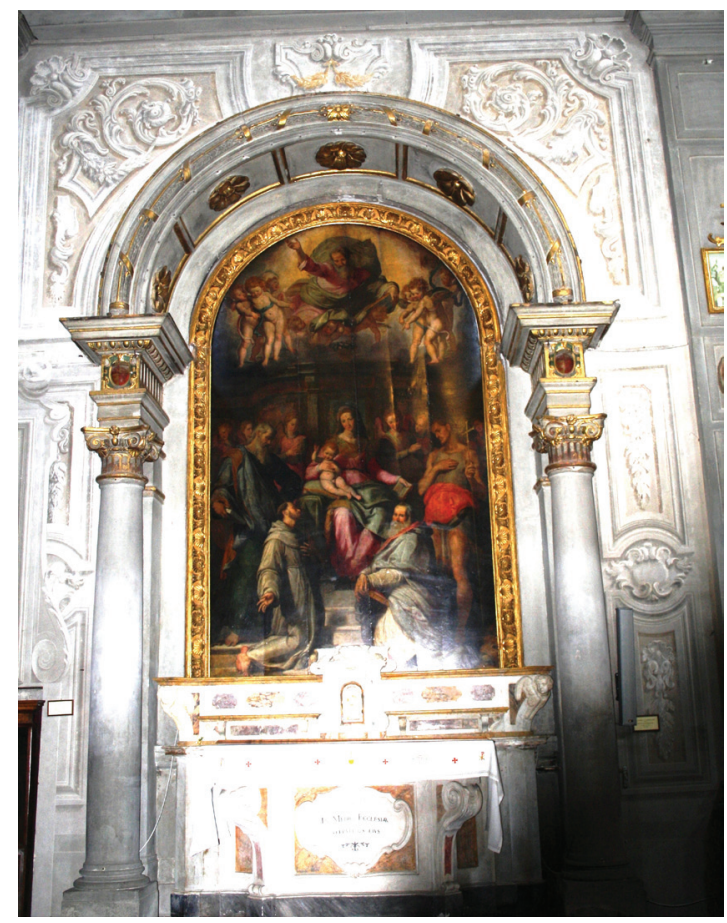

Fig. 3. Santi di Tito, Sacra Conversazione, 1565, Altar de "San Gerolamo", Iglesia de Ognissanti (Florencia).

servicio ejerció importantes cargos públicos. Primero fue paje, camarero y copero personal de Cosme I desde $1547^{6} \mathrm{y}$, años más tarde, gentiluomo de cámara del duque convirtiéndose en fiel consejero y acompañante del duque en todas las ocasiones relevantes. Don Antonio acompañó al duque durante la contienda de Siena y más tarde, con ocasión de la concesión del título de Gran Duque de Toscana en Roma (1570), asistió en calidad de mayordomo. La influencia del español era tal por entonces que podía presumir de ubicar a miembros de la comunidad española en los puestos más destacados de la milicia florentina ${ }^{7}$.

Don Antonio Ramírez de Montalvo fue patrono de la capilla mayor de la iglesia conventual de Santa Maria degli Angeli. Activa en 1578, Don Antonio encargó al

6 ASFi, Manoscritti, 321. (Arrolati della corte di Toscana dal 1540 in poi), f.29 y ss.

7 En una relación fechada en 1570 y dirigida al Católico a Madrid (localizada por la profesora M. Aglietti: AGS, Estado, 1329, ins. 137, "Relacione de los que se entiende de Florentia"), se recordaba como en dos de las tres fortalezas toscanas en posesión de los Médicis, pero datadas de presidios españoles, los castellanos eran parientes cercanos del Ramírez (uno era el hermano y otro el cuñado) y también en la tercera fortaleza habría colocado a otro de sus hombres. AGLIETTI, Marcella, "Nobili e cavalieri di Santo Stefano tra Toscana e Spagna a metà del XVIII secolo", en Quaderni Stefaniani, n XX, 2001, p. 118. 
florentino Alessandro Allori la Incoronazione della Vergine $(1593)^{8}$. La pintura alloriana, una de las obras más destacadas en la trayectoria del pintor, fue concebida con dimensiones espectaculares para ocupar un lugar privilegiado en el seno de la iglesia. La elección de la temática por parte de Antonio Ramírez de Montalvo, así como el referente iconográfico directo para Allori, fue la titularidad de la iglesia: la Madonna Regina degli Angeli.

En el campo de la milicia, durante los primeros años del Principato (1537-1542) las fortalezas de Florencia, Pisa y Livorno están bajo las órdenes de guarniciones españolas. Estos militares participan activamente en las cuestiones de la ciudad como representantes del Emperador y muchos de ellos, tras el paso de la jurisdicción de las fortalezas al duque de Florencia, permanecerán bajo su mando organizando el nuevo ejército ducal.

El español más destacado de la milicia florentina fue Don Antonio Aldana, patrono del altar de San Gerolamo en la iglesia florentina de Ognissanti (fig. 3). Este altar constituye uno de los ejemplos más interesantes del mecenazgo español en Florencia, ya que no solo es muestra del poder alcanzado por la familia Aldana en el entourage de los Médicis, sino que simboliza un cambio en la evolución pictórica de la escuela florentina con el encargo de la Sacra Conversazione a Santi di Tito. La pintura es un "manifiesto" de la pintura florentina dentro del programa estilístico de Santi di Tito, en el que el pintor ensalza la simplicidad formal y el genuino sentimiento religioso frente a los caracteres manieristas. La obra presenta, tanto en el esquema compositivo como en el contenido, la recuperación de la tradición del primo Cinquecento, una de las constantes de los pintores "riformati" quienes, impulsados por el ánimo de dotar pureza e inteligibilidad a las formas y al contenido, recuperan la tradición pictórica del primer Renacimiento.

En la misma iglesia de Ognissanti, el altar dedicado a la Inmaculada Concepción fue comisionado por el español Orlando Tapia hacia 1580. En esta ocasión, la pintura que decoró el altar fue la Allegoria dell'Immacolata Concezione, firmada y fechada en 1566 por Carlo Portelli. Es, sin duda, una obra clave en la trayectoria del artista, no sólo porque constituye un hito en la evolución estilística de Portelli (es la obra por excelencia dentro del periodo de madurez del pintor), sino porque desde un punto de vista temático se ha convertido en una de las pinturas más atractivas de la segunda mitad del quinientos por la compleja iconografía que contiene.

En lo que respecta a la presencia española en el ámbito religioso, las gestiones de la duquesa Leonor de Toledo permitieron el asentamiento de la Compañía de Jesús en la ciudad del Arno en 1546, momento a partir del que los grandes duques dedican sus esfuerzos en favorecerla por encima de otras órdenes religiosas, cediéndole el uso de una antigua iglesia y las casas contiguas para construir, bajo los dictados de Bartolommeo Ammannati ${ }^{9}$, el nuevo Collegio junto al prestigioso Palazzo Medici de Via

\footnotetext{
8 Óleo sobre tabla (415x 246,2 cm.). Florencia, Galleria dell'Accademia. Inv. 1890 n. 4630. Situada en el lateral derecho de la Tribuna del David desde 1983, después de haber sido recuperada del Convento de San Marcos.

9 HURX, Merlijn, "Bartolomeo Ammannati and the College of San Giovannino in Florence. Adapting Architecture to Jesuit Needs", en Journal of the Society of Architectural Historians, vol. 68, n 3, 2009, pp. 338-357.
} 


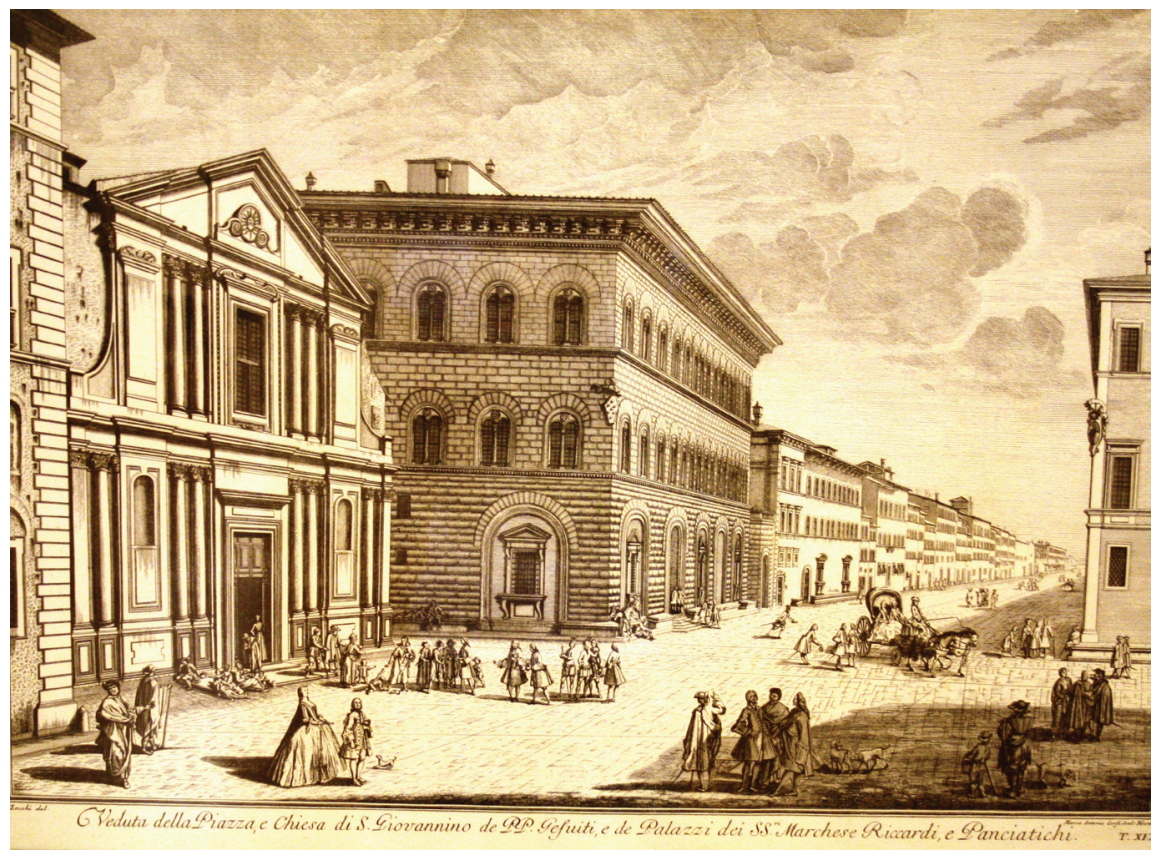

Fig. 4. Giuseppe Zocchi, Veduta della Piazza e Chiesa di S. Giovannino de PP. Gesuiti e de Palazzi dei SS ${ }^{r i}$ Marchese Riccardi e Panciatichi, 1744, Museo Firenze Com'era (Florencia).

Larga, símbolo del poder mediceo por entonces (fig. 4). Por otro lado, en 1562 Cosme I de Médicis funda la Orden de Caballeros de Santo Stefano ${ }^{10}$, en la que serán investidos caballeros ochenta españoles residentes en Florencia hasta el final del siglo.

En el ámbito académico, un español fue nombrado rector de la Universidad de Pisa (regenerada en 1543 como universidad estatal), como representante de un gran número de docentes y alumnos españoles en dicha sede universitaria, reconvertida por Cosme I de Médicis en la universidad oficial del gran ducado.

Sin duda, la presencia de mercaderes españoles es el aspecto más interesante de los que constituyen las principales arterias del estado florentino (corte, milicia, religión, universidad y mercado), ya que en éstos constituyen el origen de la comunidad española de la Florencia del Renacimiento.

10 Para tener una idea general de la Orden de Santo Stefano, véanse las actas del siguiente congreso: AGLIETTI, Marcella (coord.), Istituzioni, potere e società. Le relazioni tra Spagna e Toscana per una storia mediterranea dell'Ordine dei Cavalieri di Santo Stefano (Convegno Internazionale di Studi, Pisa, 2007), Pisa, ETS, 2007. La integración de los españoles en la nobleza toscana se produjo gracias a la inscripción en la Orden de San Esteban; desde su puesta en marcha (1562) numerosos españoles solicitaron su admisión, alzándose como el colectivo extranjero más numeroso. Éste fue el caso de los miembros de la familia Aldana, los Ramírez de Montalvo, los Suárez de la Concha y los Ximénez de Aragón. Ingresar en la orden fue el paso previo para formar parte de los Libri d'oro, donde también se registraron otras familias españolas que llegaron a Florencia en el siglo XVII, como los Narváez Saavedra. 
Actuando en calidad de factores de los socios asentados en territorio hispano, los mercaderes castellanos fueron protagonistas de un intenso tráfico comercial BurgosFlorencia ${ }^{11}$ y sentaron las bases del Consulado español de la capital toscana en 1494. Nacía, de este modo, una joven comunidad de mercaderes que la historiografía reconoce como "factoría" 12 y a la que la documentación se refiere como "nazione spagnola"13; una realidad social que debe su razón de ser a la hábil política mercantil puesta en marcha por los Reyes Católicos en las postrimerías del siglo $\mathrm{XV}^{14}$.

La "nazione spagnola" funcionó bajo la dirección del Consulado de Burgos y formó parte de un sistema de "naciones" que tenían sede en las principales plazas europeas ${ }^{15}$. Como parte del sistema, la "nazione spagnola" de Florencia alcanzó un alto grado de desarrollo organizativo por parte del comercio y de los mercaderes castellanos que, asentados en la capital toscana, hicieron de la ciudad una de las más desarrolladas a lo largo del quinientos. La ciudad del Arno no sólo constituía un enclave estratégico debido a su situación geográfica privilegiada, sino que además presentaba una serie de posibilidades que, como mercado de fuerte potencial, abría al tráfico español hacia otros centros italianos.

Esta fue la oportunidad que les permitió establecerse y residir en Florencia durante más de un siglo ${ }^{16}$, periodo en el que no se consideraban extranjeros en la ca-

11 HOSHINO, Hidetoshi, L'arte della lana in Firenze nel Basso Medioevo. Il comercio della lana e il mercato dei panni fiorentini nei secoli XIII-XV, Firenze, Olschki, 1980, p. 278. Sólo después de 1485 la lana castellana, que no había aparecido nunca en el mercado florentino, empezó a superar en calidad a la italiana, procedente del Abruzzo.

12 COLMEIRO, Manuel, Historia de la economía politica en España, Madrid, Taurus, 1965, p. 908; AGLIETTI, Marcella, "Patrizi, cavalieri e mercanti. Politiche di nobiltà tra Toscana e Spagna in Età Moderna", en AGLIETTI, Marcella (coord.) (2007), op. cit., p. 348.

13 Sobre la definición de nazione, véase la que ofrece el investigador Petti Balbi: "Le nazione mercantili sono associazioni privati e volontarie, poste in essere da uomini d'affari che soggiornano temporaneamente in terra straniera, che si coagulano sulla base della provenienza, per perseguire interessi e obiettivi comuni e che si propongono come diretti interlocutori dei poteri locali, senza allentare $i$ vincoli con la madrepatria o cercare sistemazione in zone esclusive. Nascono in ambito mercantile, come del resto le Mercanzie cittadine, ma assumono subito una valenza più complessa perché, per esistere, la natio ha bisogno anche di un supporto esterno, di un riconoscimento politico e giuridico. Occorre cioè, che la città d'appartenenza sia disposta a tutelar il complesso di interessi privati posti in essere dai propri mercanti nel paese straniero e che a sua volta la autorità di quest'ultimo, in deroga al principio dell'esercizio delle funzioni di diritto pubblico all'interno del loro territorio, permettano alle comunità forestiere di essere regolate da leggi e da magistrati propri riconosciuti dalla madrepatria (...)". PETTI BALBI, Giovanna, "Le nationes italiane all'estero", en Il Rinascimento italiano e l'Europa, vol. IV, FRANCESCHINI, Franco, GOLDTHWAITE, Richard A., MUELLER, Reinhold C (coord.), Commercio e cultura mercantile, Vicenza, Fondazione Cassamarca, 2007, p. 399. Por su parte, Richard Goldthwaite define el concepto de "nación" como "an association sep up to handle commercial and juridical disputes among its members and to deal collectively with a local government to try go gain legal status and privileges. Statutes established the parameters of its authority, and elected officials, usually a consul and two councilors, directed its affairs, perhaps aided by a notary and a treasurer". GOLDTHWAITE, Richard, The economy of Renaissance Florence, Baltimore, The Johns Hopkins University Press, 2009, p. 108.

14 Vid. BASAS FERNÁNDEZ, Manuel, El Consulado de Burgos en el siglo XVI, Madrid, CSIC, 1963.

15 Sobre este aspecto, son numerosas las publicaciones del profesor Hilario CASADO ALONSO. A modo de ejemplo, vid.: El triunfo de Mercurio. La presencia castellana en Europa (Siglos XV y XVI), Burgos, Cajacírculo, 2003.

16 DINI, Bruno, "Mercaderes españoles en Florencia (1480-1530)", en Actas del V Centenario del Consulado de Burgos 1494-1994, Burgos, Diputación Provincial, 1994, p.346. El profesor Dini analizaba 
pital toscana sino que estaban en tierra propia, tanto como las colonias de italianos en España ${ }^{17}$.

Fruto de las relaciones comerciales que durante el siglo XVI vincularon Castilla y Toscana, la "nazione spagnola" favoreció el buen funcionamiento de numerosas "sociedades" o "compañías" de mercaderes cuyos principales representantes, asentados en Castilla, encontraban salida a sus productos en Italia gracias a los factores asentados en Florencia. Para la industria florentina del momento, la presencia castellana se tradujo en una situación ventajosa para ambas partes: los españoles proporcionaban materias primas (sobre todo lana) además de comprar a los sederos florentinos productos terminados que después exportaban a España ${ }^{18}$. Por tanto, la presencia de la "nazione spagnola" contribuyó de forma irrefutable al esplendor económico de la ciudad del Arno a lo largo del quinientos.

Como sucedía con otras naciones castellanas repartidas por Europa, la "nación española" de Florencia gozaba de autonomía política ${ }^{19}$, jurisdiccional, fiscal y religiosa. Será precisamente este último aspecto (autonomía religiosa), el que justifique el mecenazgo español en la sala capitular del convento dominico de Santa Maria Novella, el Cappellone degli Spagnoli (anteriormente conocido como Capitolo di Mico Guidalotti) ${ }^{20}$.

Aunque la presencia española en este espacio se remonta, como mínimo, a $1490^{21}$, lo cierto es que la "nazione spagnola" no obtuvo la propiedad exclusiva y definitiva de la sala capitular hasta $1566^{22}$ bajo el patronato de Santiago Apóstol; sólo a partir de

el caso de los mercaderes españoles afincados en Florencia entre 1480 y 1530, asegurando que residieron en la ciudad desde aquel momento hasta el final de la centuria. Las investigaciones que he llevado a cabo me han permitido reafirmar la tesis del profesor B. Dini e incluso localizar las residencias de algunos de los mercaderes españoles que vivieron en Florencia en la segunda mitad del quinientos.

17 BASAS FERNÁNDEZ, Manuel, "El seguro marítimo sobre la vida de traficantes castellanos por el Mediterráneo en el siglo XVI”, en RAGOSTA, Rosalba (coord.), Le genti del mare Mediterraneo, vol. II, Napoli, Pironti, 1982, p. 705.

18 FUSARO, Maria, "Gli uomini d'affari stranieri in Italia", en FRANCESCHI, Franco; GOLDTHWAITE, Richard A. y MUELLER, Reinhold C. (2007), op. cit., p. 388.

19 Políticamente la colonia estaba bajo la tutela de un "cónsul", un magistrado elegido por la propia comunidad de forma independiente del monarca de Castilla, del Consulado de Burgos y de los duques de Toscana. Sobre la figura del cónsul de mercaderes vid.: FERREIRA PREGUE, Elisa, "Cónsules de castellanos y cónsules de españoles en el Mediterráneo bajomedieval", en CASADO ALONSO, Hilario (coord.), Castilla y Europa. Comercio y mercaderes en los siglos XIV, XV y XVI, Burgos, Diputación Provincial, 1995, pp. 191240.

20 El nombre se debe a que dicho capitolo fue edificado hacia 1340 por el arquitecto Fra Jacopo da Nepoziano bajo el patrocinio del mercader florentino Mico Guidalotti. Vid.: PAATZ, Walter y Elisabeth, Die kirchen von Florenz, vol. III, Frankfurt am Main, V. Klostermann, 1952, p. 720.

21 Esta es la fecha que propone el profesor Roberto LUNARDI, Arte e storia in Santa Maria Novella, Firenze, Salani, 1983, p.79. Por su parte, James WOOD BROWN retrasa la fecha a 1535. Vid.: The Dominican Church of Santa Maria Novella at Florence: A historical, architectural, and artistic study, Edimburgh, O. Schulze, 1902, p. 148. Téngase en cuenta la proximidad de esta fecha con la aparición de la primera colonia de mercaderes españoles en Florencia, lo que nos permite confirmar que desde un primer momento la "nación española" escogió el convento de Santa María Novella como espacio religioso para sus celebraciones.

22 Para ello la "nación española" dirigió una súplica al duque Cosme I de Médicis, quien actuó como mediador entre los religiosos del convento (hostiles a ceder la sala capitular) y los españoles. Sobre este aspecto, vid.: LUNARDI, Roberto, "La ristrutturazione vasariana di Santa Maria Novella", en Memorie domenicane, $\mathrm{n}^{\circ} 19,1988$, pp. 410-411. 


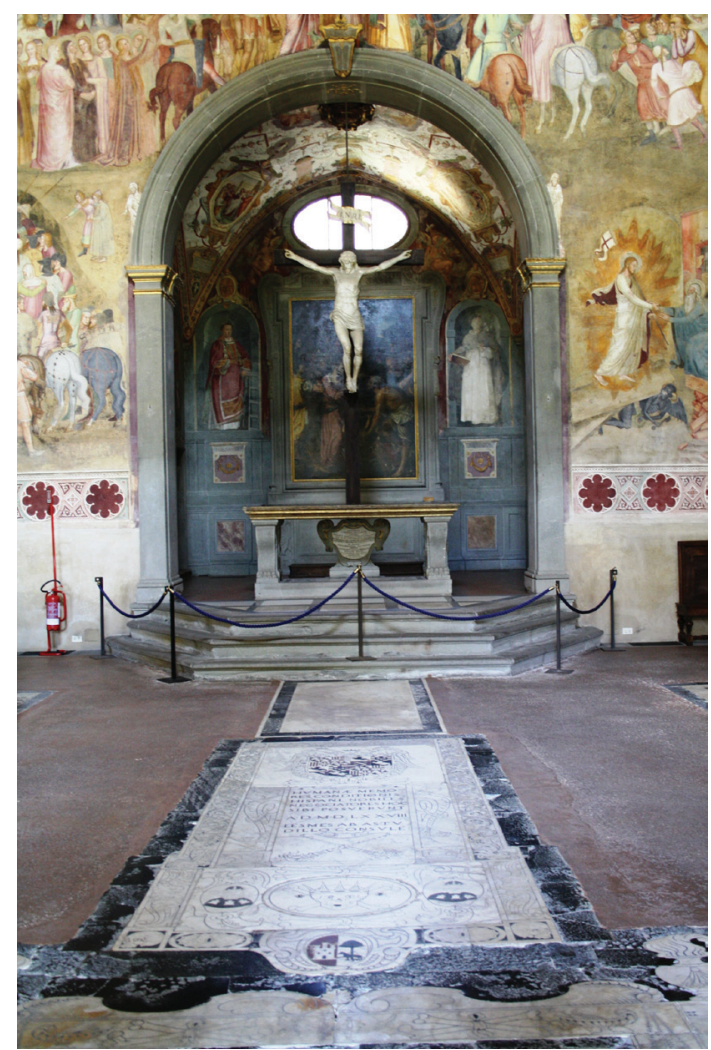

Fig. 5. Alessandro Allori y Alessandro Pieroni, Cappellone degli Spagnoli, 1566-1592, Museo di Santa Maria Novella (Florencia).

entonces, la sala capitular del convento dominico pasó a conocerse como Cappellone degli Spagnoli (fig. 5).

En 1566, Baltasar Suárez de la Concha, cónsul de la "nazione spagnola”, compró la que por entonces era la sala capitular del convento, iniciando una reforma del conjunto que terminó en 1592. Las intervenciones llevadas a cabo por los españoles en la capilla deben ser contextualizadas en el marco de una renovación que envolvió al convento dominico en un periodo de profundas transformaciones bajo los dictámenes del espíritu tridentino ${ }^{23}$. Bajo las directrices de Alessandro Allori y según una costumbre habitual de la época ${ }^{24}$, Sandrino dell'Impruneta (más conocido como

23 Sobre este aspecto, vid.: HALL, Marcia B., Renovation and Counter-Reformation. Vasari and Duke Cosimo in Santa Maria Novella and Santa Croce 1565-1577, Oxford, Clarendon Press, 1979.

24 La costumbre de pintar sobre modelos (en nuestro caso, sobre un modelo preestablecido por Allori) correspondía a un modus operandi de los talleres de la época, propia de los artistas más destacados del panorama artístico del momento como eran el mismo Alessandro Allori y Santi de Tito. Sobre este aspecto 
Alessandro Pieroni $)^{25}$ ejecutó un ciclo pictórico en el ábside de la capilla que representa una verdadera apoteosis de la catolicísima España a través de la serie dedicada a Santiago Apóstol (en la bóveda) y a seis santos españoles (en las tres paredes del ábside) ${ }^{26}$.

Junto a las intervenciones llevadas a cabo en el cappellone en el último tercio del siglo XVI, los miembros de la "nazione spagnola" de Florencia se vieron implicados en la renovación del ciclo pictórico del Chiostro Grande del convento dominico de Santa Maria Novella. El programa iconográfico diseñado para este espacio preveía la decoración de cincuenta y dos lunetas con frescos ilustrativos de la vida de Cristo, de Santo Domingo y de los principales santos de la Orden. El género de los temas, así como la finalidad de los mismos, hacían casi imperativa la referencia a la más antigua tradición florentina del fresco narrativo anterior a la Maniera ${ }^{27}$.

En la decoración del nuevo ciclo pictórico participaron la mayor parte de los pintores de la generación posvasariana ${ }^{28}$. Muchos de ellos, como Alessandro Allori, Giovan Maria Butteri, Alessandro Fei o Santi di Tito, se habían reafirmado en el panorama artístico florentino de la segunda mitad del siglo trabajando en el seno de la corte medicea. Otros, como Bernardino Poccetti, Ludovico Buti, Benedetto Veli, Gregorio Pagani y Ludovico Cigoli, habían tomado del magisterio de estos el impulso necesario para desarrollar sus propias carreras.

Los frescos comisionados por los españoles, repartidos a lo largo de las galerías sur y oeste del claustro y realizados entre 1582 y 1584 , son aquellos que tienen como protagonista al fundador de la orden dominica, Santo Domingo de Guzmán, a excepción del situado en el ángulo sureste, que pertenece al ciclo dedicado a la vida de Cristo. La razón que explica la selección del ciclo dedicado a la vida de este santo por parte de los españoles está vinculada al hecho de que el santo era oriundo de Careluega en la provincia de Burgos, ciudad de la que procedían la mayor parte de los mercaderes españoles que comisionaron estos frescos.

Especialmente significativo por la temática que representa es el fresco de La liberación de los peregrinos en una tempestad de mar gracias a la intercesión de Santo Domingo, ya que representa un episodio poco frecuente de la hagiografía dominica ${ }^{29}$.

véase: LECCHINI GIOVANNONI, Simona y COLLARETA, Marco, Disegni di Santi di Tito, 1536-1603, Firenze, L.S. Olschki, 1985, p.11.

25 Sobre Alessandro Pieroni vid.: LECCHINI GIOVANNONI, Simona, "Per Alessandro Pieroni: una proposta per la decorazione cinquecentesca dell'abside del cappellone degli spagnoli in Santa Maria Novella a Firenze", en Studi di Storia dell'Arte, nº 2, 1991, pp. 321-338.

26 La presencia castellana en el Cappellone degli Spagnoli, así como el análisis iconográfico del conjunto pictórico está analizado en: GONZÁLEZ TALAVERA, Blanca, "Imagen y poder español en la Florencia medicea: La Capilla de los Españoles de Santa María Novella", en Actas de la XI Reunión Científica de la Fundación Española de Historia Moderna, Granada, Editorial Universidad de Granada, vol. I, 2012, pp. 361-372.

27 FREEDBERG, Sidney Joseph, Pintura en Italia 1500-1600, Madrid, Cátedra, 1983, p. 627.

28 En el caso que nos interesa, los artistas comisionados por los españoles fueron: Bernardino Pocetti, Santi di Tito, Giovanni Maria Butteri y Alessandro Fei.

29 KAFTAL, George, St. Dominic in early Tuscan painting, Oxford, Blackfriars, 1948, p. 13. 
Comisionado por Lesmes de Astudillo ${ }^{30}$ (cónsul de la "nación española" de Florencia en aquel periodo) y ejecutado por el pintor Santi di Tito, el fresco recoge el momento en el que el santo, en el camino hacia Santiago de Compostela, socorre a un grupo de peregrinos que intenta salvarse de las aguas de un río cercano a Tolosa, ciudad en la que Santo Domingo debía fundar su primer convento.

Se deduce, pues, que los miembros de la comunidad española de Florencia constituyen uno de los pilares básicos en la construcción del Principato con su presencia en las principales arterias de este nuevo estado y en el mecenazgo de la capital toscana. El análisis de algunas de las manifestaciones artísticas comisionadas por este colectivo invita a continuar el estudio de su mecenazgo.

30 Sobre este ilustre mercader, vid:: GONZÁLEZ TALAVERA, Blanca, "Mecenazgo español en Florencia: Lesmes de Astudillo y la villa de Montughi (1589-1592)", en Cuadernos de Arte e Iconografia, n 38, 2010, pp. 405-428. 